\title{
Electromagnetic Guidance Control System of Rail Vehicle Driven by Linear Motor
}

\author{
Koichi MATSUOKA \\ Chief Engineer, Electric Drive Systems G., \\ Vehicle Technology Development Div., \\ Technological Development Dept.
}

\begin{abstract}
We are now making research and development efforts on a new railway system which is driven by linear motors, supported by iron wheels, and guided by electromagnets. The guidance force is produced by electromagnetic attraction between on-board electromagnets and track-side guide rails. As one step in the development, we examined the performance of the electromagnetic guidance control system by using a rotary disc type experimental facility. Frictional force between the supporting iron wheel and rail may affect the guidance control system as disturbance. To compensate for the non-linearity and modeling errors of the frictional force, we introduced a disturbance observer and feed forward control method. Kalman filter which estimates state variables was also used with LQI control to obtain satisfactory performance. This paper presents the system configuration and experimental results.
\end{abstract}

Keywords : linear motor drive, electromagnetic guidance control, friction, optimum regulator.

\section{Introduction}

Linear motor drive features advantages of higher speed, higher acceleration and operation at steeper grades when compared with the conventional traction system, which depends on the adhesive force between wheel and rail. So various types of guided transportation systems driven by linear motor have been investigated all over the world.

As one of the new guided transportation systems for the next generation, we are examining a system driven by a permanent magnet linear synchronous motor, supported by iron wheels, and guided by electromagnetic suspension. Fig. 1 shows the configuration of

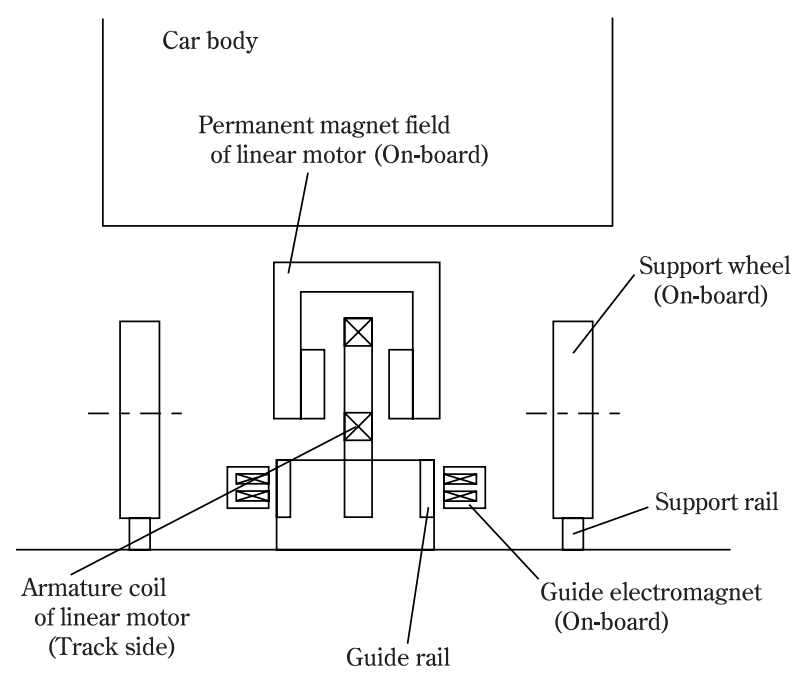

Fig. 1 Scheme of linear motor driven, iron wheel supported and electromagnetically guided rail vehicle. this system. This system has separated elements for traction, suspension, and guidance. Therefore, each of the elements can be designed to its optimum point independently. But, the frictional force between support wheel and rail may disturb the electromagnetic guidance control system. As the first step for development, we constructed an experimental electromagnetic guidance control system including friction device, and performed some experiments.

\section{Structure of the Experimental Facility.}

Fig. 2 shows the structure of the experimental facility. The iron disc, which corresponds to the trackside guide rail is $1730 \mathrm{~mm}$ in diameter, and $390 \mathrm{~mm}$ in thickness, and rotates at the maximum peripheral speed of $300 \mathrm{~km} / \mathrm{h}$. Guide magnets are attached to a supporting arm, which is $2200 \mathrm{~mm}$ long from the pivot to the center of the guide magnet. The supporting arm can swing around the pivot, and has an additional friction device at the end. Adjustable weights are mounted on the supporting arm to adjust the equivalent moving mass. The equivalent mass at the magnet center is $285.2 \mathrm{~kg}$. This is about one-sixteenth the full scale vehicle. The iron disc is driven by a D.C. motor connected to the axle. Fig. 3 shows the geometry of the guide electromagnet. A race-track-shaped field coil is attached to the Eshaped iron core. Two electromagnets are placed at the supporting arm to face each other with the rotating iron disc between. Fig. 4 shows the cross-section of the experimental facility. Excitation current of the electromagnet is fed from a PWM-controlled IGBT chopper. Fig. 5 shows the appearance of the experimental facility. 


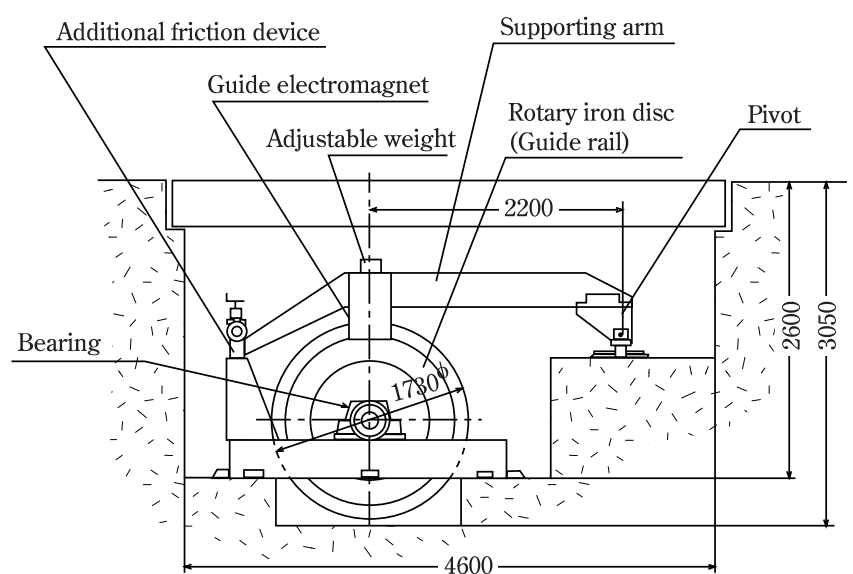

Fig. 2 Structure of rotary disc type experimental facility for electromagnetic guidance control.

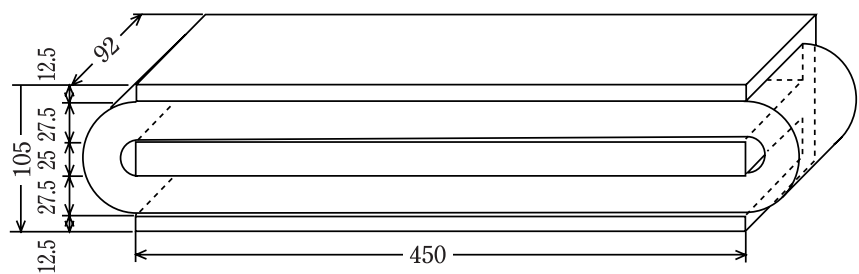

Fig. 3 Structure and dimension of guide electromagnet

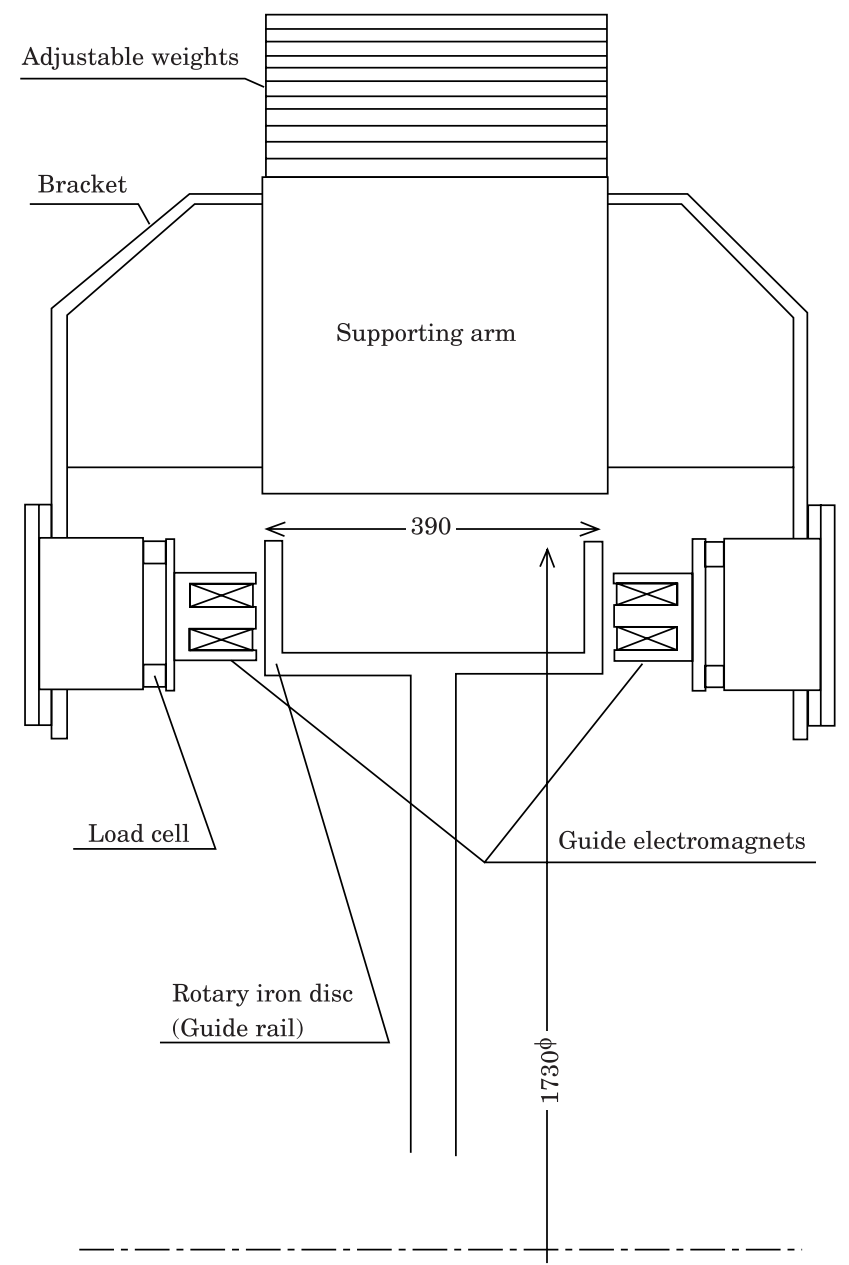

Fig. 4 Cross-section of rotary disc type experimental facility

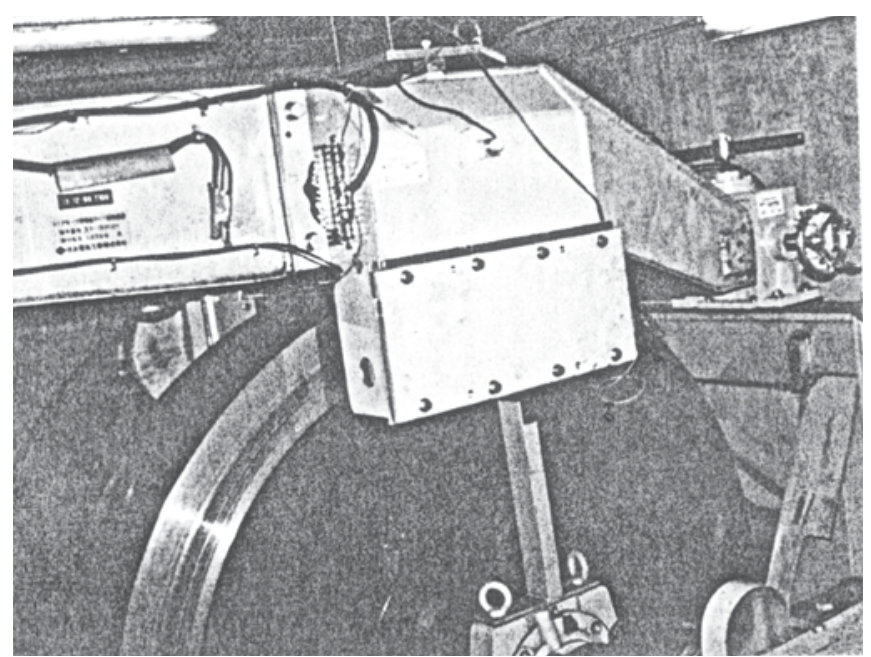

Fig. 5 Appearance of experimental facility

\section{Configuration of the Control System}

Fig. 6 shows the configuration of the electromagnetic guidance control system. When the magnets are placed at the reference position, both gaps between the faces of right and left electromagnets and guide rails are identical. At the reference position, currents of the same level are fed to both magnets, and their electromagnetic forces are balanced. In this experimental system, the attractive forces of electromagnets are controlled to maintain the gap between the electromagnets and the

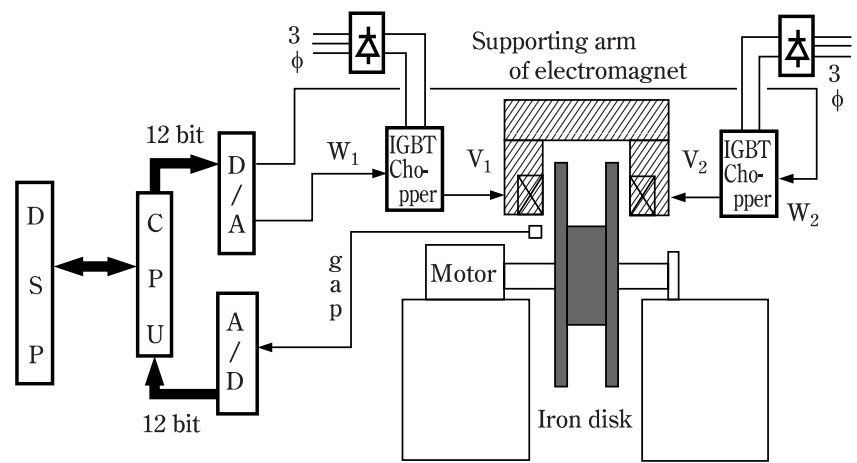

Fig. 6 Configuration of experimental electromagnetic guidance control system

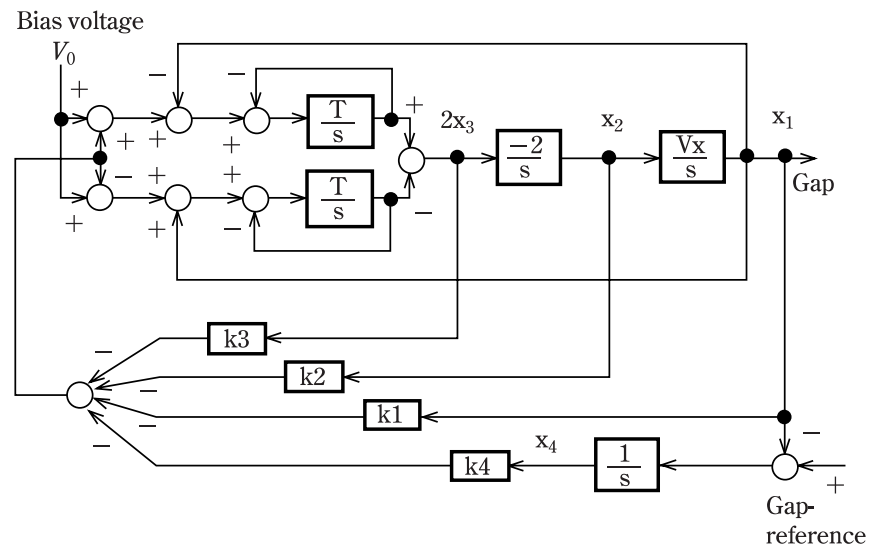

Fig. 7 Block diagram of electromagnetic guidance control system using LQI 
guide iron disc constant under the presence of disturbance. A block diagram of this control system is shown in Fig.7. An eddy current type sensor was used to detect the gap between one of the electromagnets and the iron disc. The controller includes a DSP (TMS320C25) and $12 \mathrm{bit} \mathrm{A} / \mathrm{D}, \mathrm{D} / \mathrm{A}$ converters. The sampling period is $2 \mathrm{~ms}$. The power amplifier for each electromagnet is a IGBT (600V, 50A) chopper.

\section{Control Method}

We selected displacement, velocity, acceleration, and integral of setting errors as the state variables. This guidance control system aimed at controlling the attractive force of the electromagnets with high non-linearity. The state feedback control (LQI control method) was used. The designed response time was $0.1 \mathrm{~ms}$. For this system, the disturbance estimation observer and the feed forward method were applied to compensate for the modeling errors due to friction and other external forces. In addition, the state variables were estimated with a Kalman filter. The proposed control system was a combination of these methods.

\section{Mathematical Model of the System}

The system shown in Fig. 7 is mathematically represented by equations (1) to (4). The left and right electromagnets are represented by subscripts 1 and 2 , respectively.

$$
\begin{aligned}
& \frac{d x}{d t}=v \quad \ldots \ldots \cdot \ldots \cdot \ldots \cdot(1) \\
& \frac{d v}{d t}=-\frac{k_{M}}{M}\left\{\frac{I_{1}{ }^{2}}{(X+x)^{2}}-\frac{I_{2}{ }^{2}}{(X-x)^{2}}\right\} \quad \cdots \cdot \cdot(2) \\
& \frac{d \Phi_{1}}{d t}=-R I_{1}+u_{1} \quad \text {. } \cdot \cdots \cdot \cdot \cdot \cdot \cdot(3) \\
& \frac{d \Phi_{2}}{d t}=-R I_{2}+u_{2} \quad \text {. . . . . . }(4) \\
& \Phi_{1}=L_{1} I_{1} \quad \cdot \cdot(5) \quad \Phi_{2}=L_{2} I_{2} \quad \cdots(6)
\end{aligned}
$$

Where, $X$ : Reference gap ( $8 \mathrm{~mm}$ ) $x$ : Lateral displacement $v$ : Velocity of electromagnet $I_{1}, I_{2}:$ Excitation current $u_{1}, u_{2}$ : Input voltage $\Phi_{1}, \Phi_{2}:$ Flux linkage, $M:$ Mass, $L_{1}, L_{2}$ : Inductance of electromagnet $k_{M}:$ Attraction coefficient $R$ : Winding resistance

Here, we assume that the inductance of the electromagnet is inversely proportional to the gap length.

$$
L_{1}=\frac{L}{(X+x)} \quad \cdot \cdot(7) \quad L_{2}=\frac{L}{(X-x)} \cdot \bullet \cdot(8)
$$

Where, $L$ : Constant relating to inductance of the electromagnet

By substituting the equations (5) (8) into the equations $(2) \sim(4)$, we get the following equations.

$$
\begin{aligned}
& \frac{d v}{d t}=-\frac{k_{M}}{M L^{2}}\left\{\Phi_{1}^{2}-\Phi_{2}^{2}\right\} \quad \cdots \cdot \cdots \cdot(9) \\
& \frac{d \Phi_{1}}{d t}=-\frac{R}{L} \Phi_{1}(X+x)+u_{1} \quad \ldots . \cdot \cdots \cdot(10) \\
& \frac{d \Phi_{2}}{d t}=-\frac{R}{L} \Phi_{2}(X-x)+u_{2} \quad \text {. . . . } \cdot(11)
\end{aligned}
$$

After normalization and linearization of these state variables around their equilibrium points, we get the following nominal state space equation (12).

$$
\begin{aligned}
& \frac{d x}{d t}=A x+B u \\
& x=\left[\begin{array}{l}
x_{1} \\
x_{2} \\
x_{3}
\end{array}\right] \quad A=\left[\begin{array}{lll}
0 & V_{x} & 0 \\
0 & 0 & -4 \\
-T & 0 & -T
\end{array}\right] \quad B=\left[\begin{array}{l}
0 \\
0 \\
T
\end{array}\right] \\
& V_{x}=\frac{k_{M} \Phi_{0}{ }^{2}}{X M L^{2}} \quad, \quad T=\frac{R X}{L}
\end{aligned}
$$

Where, $\Phi_{0}$ : Flux linkage at the reference gap We selected state variable as follow :

$$
\begin{aligned}
& x_{1}=g \\
& x_{2}=q \\
& x_{3}=\frac{1}{2}\left(\phi_{1}-\phi_{2}\right) \\
& u=\frac{1}{2}\left(w_{1}-w_{2}\right)
\end{aligned}
$$

where, $g$ : Normalized displacement

$$
\begin{aligned}
& q: \text { Normalized velocity } \\
& \phi: \text { Normalized flux linkage } \\
& w: \text { Normalized voltage }
\end{aligned}
$$

The state variables were displacement from the reference gap length $8 \mathrm{~mm}\left(x_{1}\right)$, velocity $\left(x_{2}\right)$ and half of the flux difference between the left and right electromagnets $\left(x_{3}\right)$. The input variable was half of the voltage difference $(u)$.

\section{LQI Control}

We designed an integral type optimal regulator, LQI controller, to eliminate steady state errors. The feedback gain $k$ to satisfy the required response was determined by solving the Riccati's equation. Fig. 7 shows a block diagram of the LQI control system. Simulation results of step response by using the LQI controller are shown in Fig. 8. All state variables were normalized. The gap reference was changed by $3 \mathrm{~mm}$ from the reference value $8 \mathrm{~mm}$. 

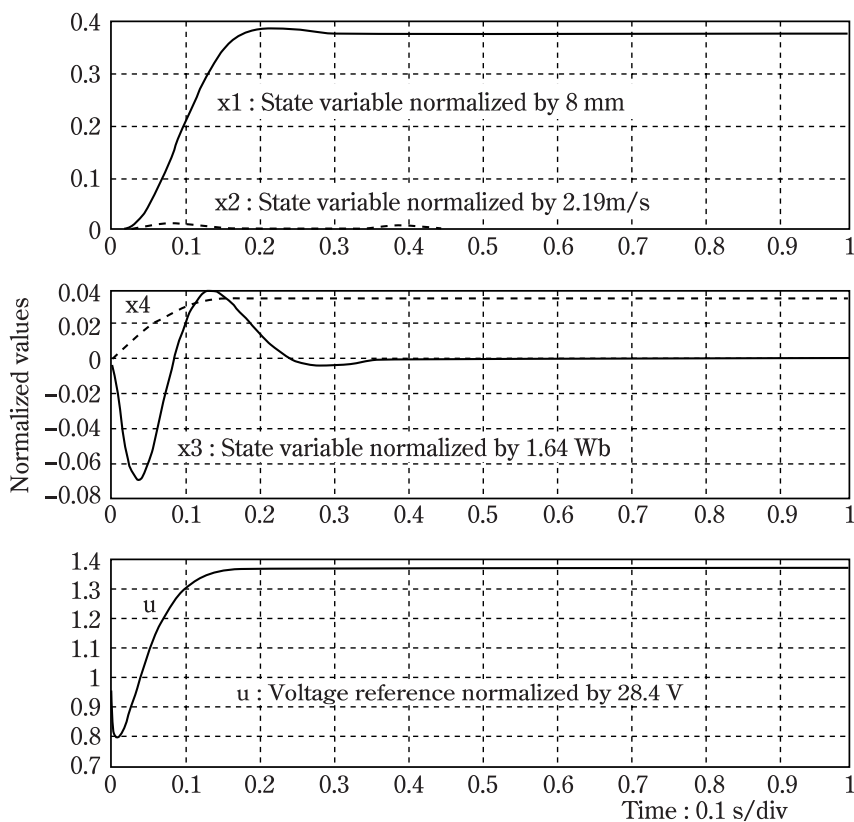

Fig. 8 Simulation result of gap response with LQI control system

\section{State Estimation Method using the Kalman Filter}

It is difficult to directly detect the state variables except the gap displacement that can be obtained with a sensor. Therefore, these variables were estimated with the Kalman filter composed of a discrete system.

The state space equation (12) is transformed into a discrete system as follows.

$$
A_{d}=e^{A \cdot T_{s}} \quad B_{d}=\int_{0}^{T_{s}} e^{A \cdot \tau} d \tau \cdot B
$$

Where, $T_{s}$ : Sampling time $(2 \mathrm{~ms})$

Then, we designed the gain $L$ to estimate the state variables which are updated according to the equation (13).

$$
\begin{aligned}
& \hat{x}_{(n+1)}=x_{(n)}+L \cdot\left(y_{(n)}-C_{d} \cdot x_{(n)}\right) \quad \cdots \cdot \cdots \cdot(13) \\
& x_{(n+1)}=A_{d} \cdot \hat{x}_{(n)}+B_{d} \cdot u_{(n)} \quad C_{d}=\left[\begin{array}{lll}
1 & 0 & 0
\end{array}\right]
\end{aligned}
$$

Where, $\hat{x}$ is the estimated state variable.

The Kalman gain $L$ was designed to make the estimated value quickly converge. The covariances $Q$ of process noise and $R$ of measurement noise were set at diag. [1. 1. 2500] and 1, respectively.

\section{Compensation for Modeling Error (Problem of Friction)}

Generally speaking, it is difficult to obtain exact state variables by using the Kalman filter when the system contains modeling errors such as frictional force, because the Kalman filter depends on a linearized model. Therefore, we did not include the frictional force of uncertain nature into the model, and treated it as a disturbance. Then we introduced a combination of disturbance esti-

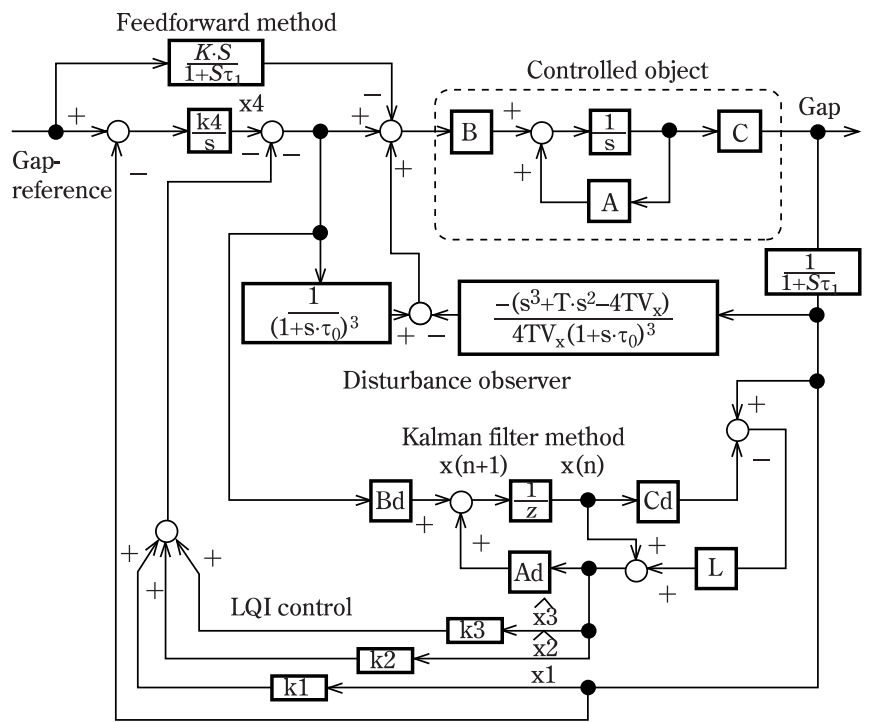

Fig. 9 Configuration of the proposed method

mation observer and feed forward compensation to compensate for the modeling errors due to frictional force. The feed forward circuit integrates the gap reference to compensate for the transitional effect from static friction to dynamic friction. Fig. 9 shows a block diagram of the proposed electromagnetic guidance control system that was the above mentioned methods.

\section{Simulation Result}

Based on the previous test results, we set a friction model shown in Fig. 10, and we simulated a step response of the system when the gap reference was changed from $8 \mathrm{~mm}$ to $11 \mathrm{~mm}$. In the case of LQI control with the designed gain, stable response was not achieved. Fig. 11 shows the simulation results of gap response of the proposed control method at the designed gain $k=[-11.27$, $-112.52,8.52,111.80]$, compared with the designed gap response of a continuous LQI control system of linear model. As this result shows, the modeling error and the influence of detecting filters of state variables were com-

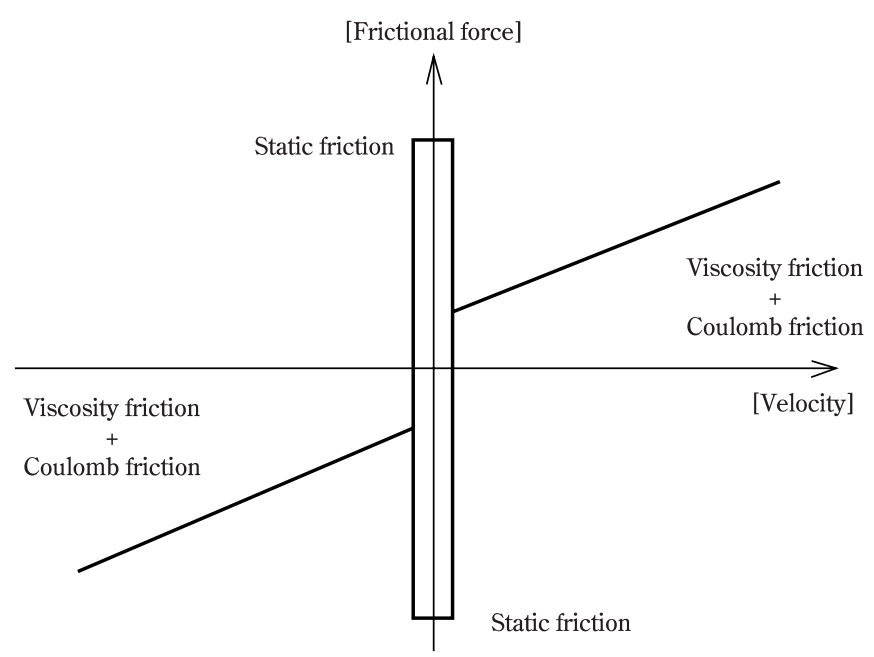

Fig. 10 Model of friction 


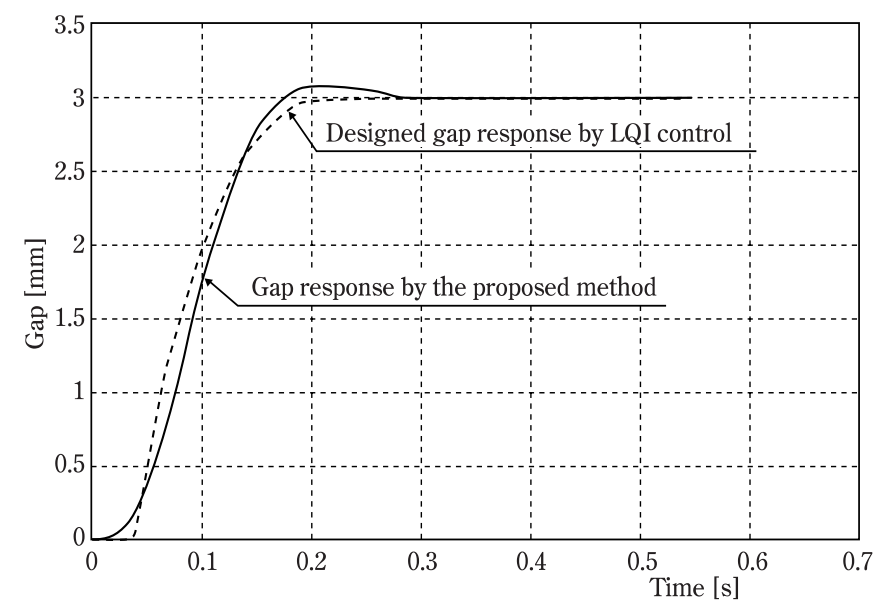

Fig. 11 Simulation result of step response

pensated for by the proposed method, and the response was close to the designed response. Using the experimental facility mentioned above, we made step response tests of the proposed control system to confirm its performance.

\section{Experimental Results}

The control system was implemented on a DSP shown in Fig. 6. Table 1 shows the parameters of the experimental system. The gains of the LQI control feedback, the disturbance observer, and the Kalman filter were set to the same values as those used in the simulation. Fig. 12 shows experimental results of the proposed system. These are the gap, the input voltage, and the current, when the gap reference was changed stepwise from $8 \mathrm{~mm}$ to $11 \mathrm{~mm}$. The response time was about $0.1 \mathrm{~ms}$, which meets the target response time.

\section{Conclusion}

To solve the influence of the modeling errors and the filters used to detect state variables, a composite control system of LQI control, the Kalman filter, disturbance observer, and feed-forward compensation system was constructed. And this system successfully attained a response close to the response characteristic designed in the LQI control system under the design feedback gain $k$.

We are grateful to Meidensha Co. and related persons for their cooperation with this study.
Table 1 System parameters

\begin{tabular}{|l|l|}
\hline Reference gap $(x)$ & $8 \mathrm{~mm}$ \\
\hline Excitation current $\left(I_{0}\right)$ & $5.48 \mathrm{~A}$ \\
\hline Input voltage $\left(V_{0}\right)$ & $28.4 \mathrm{~V}$ \\
\hline Flux linkage $\left(\phi_{0}\right)$ & $1.64 \mathrm{~Wb}$ \\
\hline Mass $(M)$ & $285.2 \mathrm{~kg}$ \\
\hline Inductance (nominal) & $0.3 \mathrm{H}$ \\
\hline Resistance $(R)$ & $5.18 \Omega$ \\
\hline$T=R \cdot X / L$ & 17.27 \\
\hline$V=k_{M} \phi_{0}{ }^{2} / M L^{2}$ & 2.19 \\
\hline$V_{X}=V / X$ & 273.5 \\
\hline Attraction coe. $\left(k_{M}\right)$ & $1.33 \cdot 10^{-3} \mathrm{~Wb} \cdot \mathrm{m} / \mathrm{A}$ \\
\hline
\end{tabular}

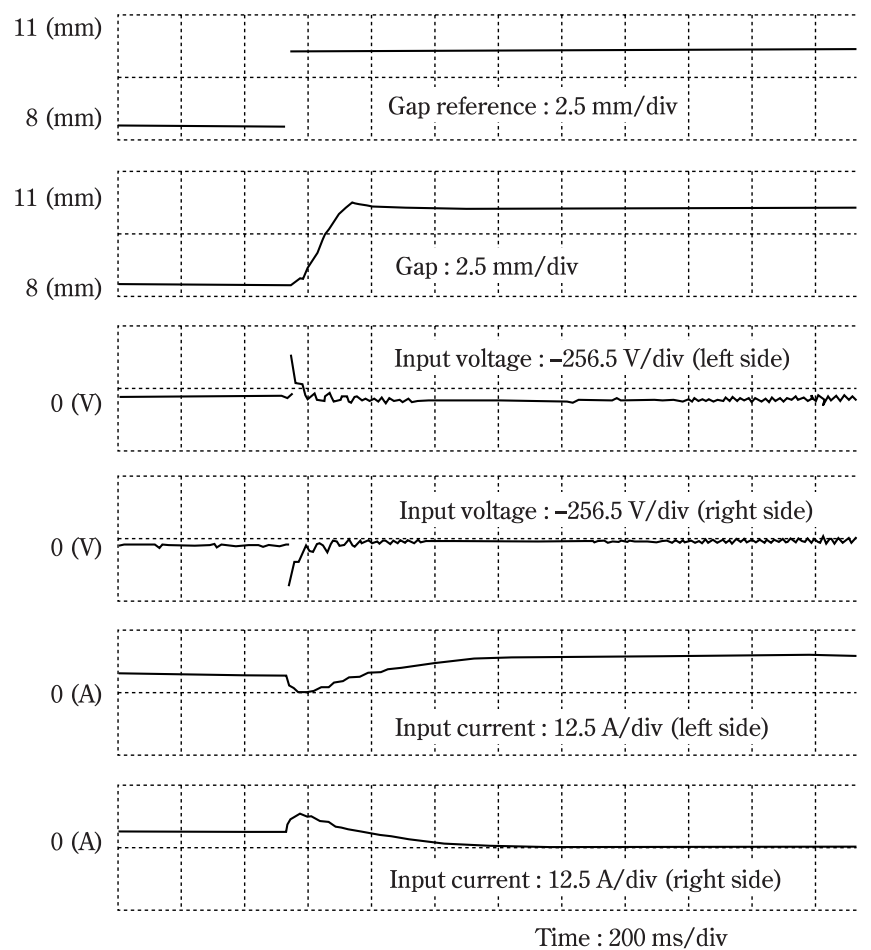

Fig. 12 Experimental result of step gap response by proposed method

\section{References}

1) Matsuoka, K., Sugimoto, T., et al. : "Trial manufacture of electromagnetic attraction guidance control test system", 1992 National Convention Record of IEE Japan, pp.7-131-7-132, 1992

2) Hori, M., Matsuoka, K., et al. : "Electromagnetic guidance control by use of Kalman filter state estimation", Proceedings of the Power Conversion Conference Yokohama 1993, pp.702-707, 1993 